\title{
Status of the ENUBET project
}

\author{
G. Brunetti* \\ INFN Sezione di Padova \\ E-mail: giulia.brunetti@pd.infn.it
}

F. Acerbi $^{a}$, G. Ballerini ${ }^{b, c}$, M. Bonesini ${ }^{c}$, C. Brizzolari ${ }^{b, c}$, M. Calviani $^{d}$, S. Carturan ${ }^{e}$, M.G. Catanesi ${ }^{f}$, S. Cecchini ${ }^{g}$, F. Cindolo ${ }^{g}$, G. Collazuol $^{h, i}$, E. Conti ${ }^{h}$, F. Dal Corso ${ }^{h}$, C. Delogu ${ }^{c, j}$, G. De Rosa ${ }^{k}$, A. Falcone ${ }^{h, i}$, B. Goddard ${ }^{d}$, A. Gola ${ }^{a}$, R.A. Intonti ${ }^{f}$, C. Jollet ${ }^{l}$, V. Kain ${ }^{d}$, B. Klicek ${ }^{m}$, Y. Kudenko ${ }^{n}$, M. Laveder ${ }^{h, i}$, A. Longhin ${ }^{h, i}$, P.F. Loverre ${ }^{o, p}$, L. Ludovici ${ }^{p}$, L. Magaletti ${ }^{f}$, G. Mandrioli ${ }^{g}$, A. Margotti ${ }^{g}$, V. Mascagna ${ }^{b, c}$, N. Mauri ${ }^{g}$, A. Meregagliaa ${ }^{q}$, M. Mezzetto ${ }^{h}$, M. Nessi $^{d}$, A. Paoloni ${ }^{r}$, M. Pari ${ }^{h, i}$, E. Parozzi ${ }^{c}, j$, L. Pasqualini ${ }^{g, s}$, G. Paternoster ${ }^{a}$, L. Patrizii ${ }^{g}$, C. Piemonte ${ }^{a}$, M. Pozzato ${ }^{g}$, M. Prest ${ }^{b, c}$, F. Pupilli ${ }^{h}$, E. Radicioni ${ }^{f}$, C. Riccio ${ }^{k, t}$, A.C. Ruggieri ${ }^{k}$, C. Scian ${ }^{i}$, G. Sirri ${ }^{g}$, M. Soldani ${ }^{b, c}$, M. Stipcevic ${ }^{m}$, M. Tenti ${ }^{g}$, F. Terranova ${ }^{c, j}$, M. Torti $^{c, j}$, E. Vallazza ${ }^{u}$, F. Velotti $^{d}$, M. Vesco ${ }^{e}$, L. Votano ${ }^{r}$

${ }^{a}$ Fondazione Bruno Kessler (FBK) and INFN TIFPA, Trento, Italy; ${ }^{b}$ Phys. Dep., Università degli studi dell'Insubria, Como, Italy; ${ }^{c}$ INFN, Sezione di Milano-Bicocca, Milano, Italy; ${ }^{d}$ CERN, Geneva, Switzerland; ${ }^{e}$ INFN Laboratori Nazionali di Legnaro, Legnaro (PD), Italy; ${ }^{f}$ INFN Sezione di Bari, Bari, Italy; ${ }^{g}$ INFN, Sezione di Bologna, Bologna, Italy; ${ }^{h}$ INFN Sezione di Padova, Padova, Italy; ${ }^{i}$ Phys. Dep. Università di Padova, Padova, Italy; ${ }^{j}$ Phys. Dep. Università di Milano-Bicocca, Milano, Italy; ${ }^{k}$ INFN, Sezione di Napoli, Napoli, Italy; ${ }^{l}$ IPHC, Université de Strasbourg, CNRS/IN2P3, Strasbourg, France; ${ }^{m}$ Center of Excellence for Advanced Materials and Sensing Devices, Ruder Boskovic Institute, Zagreb, Croatia; ${ }^{n}$ Institute of Nuclear Research of the Russian Academy of Science, Moscow, Russia; ${ }^{\circ}$ Phys. Dep. Università La Sapienza, Rome, Italy; ${ }^{p} I N F N$, Sezione di Roma 1, Rome, Italy; ${ }^{q}$ CENBG, Université de Bordeaux, CNRS/IN2P3, 33175 Gradignan, France; ${ }^{r}$ INFN, Laboratori Nazionali di Frascati, Frascati (Rome), Italy; ${ }^{s}$ Phys. Dep. Università di Bologna, Bologna, Italy; ${ }^{t}$ Phys. Dep. Università degli Studi di Napoli Federico II, Napoli, Italy; " INFN Sezione di Trieste, Trieste, Italy

The ENUBET project is studying a narrow band neutrino beam where lepton production can be monitored at single particle level in an instrumented decay tunnel and that could provide a $1 \%$ measurement of the neutrino flux at source. The three body semi-leptonic decay of kaons monitored by large angle positron production offers a fully controlled $v_{e}$ source at the GeV scale for a new generation of short baseline experiments. The ENUBET Collaboration presented at NuFact2018 the first end-to-end simulation of the beamline, the physics performance and the results of the test beam performed on the positron tagger. We reported ENUBET expected fluxes for $v_{e}$ and $v_{\mu}$.

The 20th International Workshop on Neutrinos (NuFact2018)

12-18 August 2018

Blacksburg, Virginia

* Speaker. 


\section{Monitored neutrino beams: the ENUBET approach}

Neutrino cross sections play a crucial role in the oscillation physics of the next generation $v_{\mu} \rightarrow v_{\mathrm{e}}$ experiments and are mainly limited by the estimate of the initial flux that relies on the simulation of the beam line and on the limited knowledge of the hadro-production in targets. The ENUBET project $[1,4]$ addresses this problem by developing a neutrino source based on tagging of large angle positrons from the three body semi-leptonic $\mathrm{K}_{\mathrm{e} 3}$ decays $\left(\mathrm{K}^{+} \rightarrow \mathrm{e}^{+} \pi^{0} v_{\mathrm{e}}\right)$ in an instrumented decay tunnel. This technique may lead to a reduction of the systematic uncertainties on the knowledge of the initial neutrino flux to $\sim 1 \%$, i.e. one order of magnitude lower than at present neutrino beams.

In the present ENUBET configuration the decay tunnel to be instrumented consists in an hollow cylinder with a length of $40 \mathrm{~m}$ and a $1 \mathrm{~m}$ radius. The maximum expected rate at the detector (positron tagger) is about $200 \mathrm{kHz} / \mathrm{cm}^{2}$ and $e / \pi$ separation better than $3 \%$ is needed to reject the pion background due to beam halo and to other kaon decay modes. This separation is achieved by means of longitudinally segmented calorimeters. Photons produced by $\pi^{0}$ decays are vetoed by plastic scintillator pads located just below the calorimeter. The photon veto also provides a precise timing of the positrons. These requirements constrain the detector technology, that must be based on radiation hard components with $\mathrm{O}(10 \mathrm{~ns})$ recovery time and $10 \mathrm{~cm}^{2}$ granularity.

\section{The transfer line}

The ENUBET transfer line has to focus and transport a collimated beam of pions and kaons with high efficiency, limited losses by decays and low beam-induced background. The optimization of the optics is performed with TRANSPORT [2] using a reference momentum of $8.5 \mathrm{GeV} / \mathrm{c}$ and a momentum bite of $10 \%$ while particle transport and interactions in the beamline are simulated with G4beamline [3]. Two options have been investigated: a horn-based beamline employing a pulsed horn between the target and the transport line and a static system where the transport and focusing line is implemented directly after the target and the field in the focussing or bending elements do not vary in time.

Though the horn-based solution would provide higher yields at the decay tunnel one has to consider the horn pulse limit ( $<10 \mathrm{~ms}$ ) and the tagger rate limit that would be reached with $\sim 10^{12}$ protons on target (POT) per spill. The static transfer line on the other hand only requires a single slow extraction and is beneficial in terms of pile-up effects in the tagger. Preliminary results obtained with the static option look very promising: by using a triplet-dipole-triplet scheme we obtain hadronic rates at the decay tunnel entrance that are 4 times better than the first estimate presented in [1]. Moreover, this solution would also offer the possibility of event-by-event tagging by coincidences between $v_{e}$ at a far detector and $e^{+}$at the tagger. In Tab. 1 our expected rates for the two configurations are reported. A schematic view of the static transfer line is shown in Fig. 1.

\section{The positron tagger and detectors $R \& D$}

The reference design of the positron tagger is based on calorimetric units (UCM) made of five, $15 \mathrm{~mm}$ thick, iron layers, interleaved with $5 \mathrm{~mm}$ thick plastic scintillator tiles. The total thickness 


\begin{tabular}{||c|c|c|c|c||}
\hline Focusing & $\pi^{+} /$POT $\left[10^{-3}\right]$ & $K^{+} /$POT $\left[10^{-3}\right]$ & Extraction Length & Factor w.r.t. [1] \\
\hline \hline Horn-based & 77 & 7.9 & $2 \mathrm{~ms}$ & $\times 2$ \\
\hline Static & 19 & 1.4 & $2 \mathrm{~s}$ & $\times 4$ \\
\hline
\end{tabular}

Table 1: Expected rates of $\pi^{+}$and $K^{+}$in $[6.5 \div 10.5 \mathrm{GeV} / \mathrm{c}]$ range at the decay tunnel entrance for the two possible focusing schemes. The improvement factor in kaon transport with respect to [1] is shown in the last column.

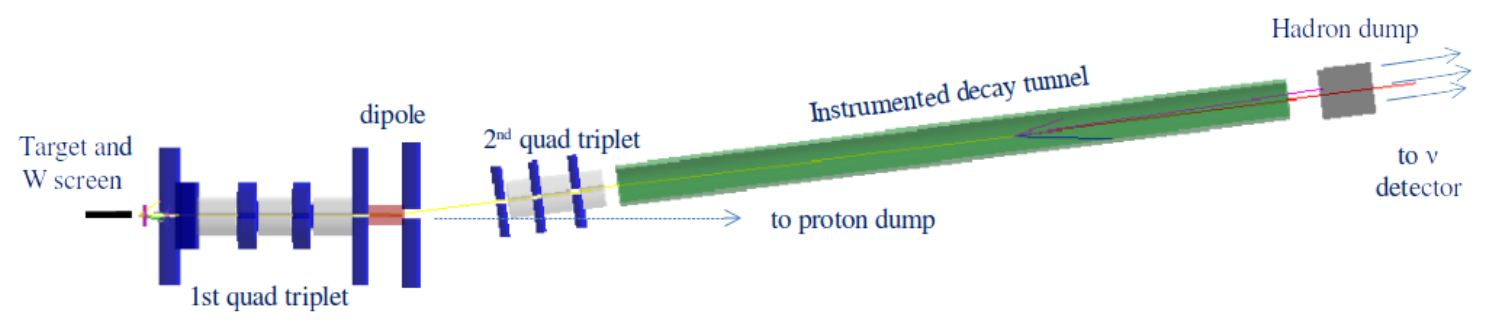

Figure 1: Schematics of the static line design.

of the UCM module corresponds to $4.3 X_{0}$ and its transverse size is $3 \times 3 \mathrm{~cm}^{2}$. The readout is performed through WLS fibers coupled to silicon photomultipliers (SiPM). Doublets of plastic scintillator tiles $\left(3 \times 3 \mathrm{~cm}^{2}\right.$ surface and $5 \mathrm{~mm}$ thickness for $\left.\sim 0.02 X_{0}\right)$ provide $e^{+} / \pi^{0}$ separation. This photon-veto also provides the absolute time of the event ( $\mathrm{t}_{0}$-layer). During 2016-2018 tests in four two-week slots of data taking were performed to validate prototypes for the $t_{0}$-layer and the calorimeters both in shashlik and lateral readout modes $[5,6,7,8]$. At present, both options fulfill the requirements of ENUBET and a final decision will be taken in 2019 based on the result of final tests in 2018.

During the lifetime of the experiment the SiPM will integrate a neutron fluence of $\mathrm{O}\left(10^{11}\right)$ $1 \mathrm{MeV}$-equivalent neutrons $/ \mathrm{cm}^{2}$. A dedicated irradiation campaign was performed in $2017 \mathrm{using}$ the $\mathrm{CN}$ accelerator at the INFN-LNL laboratories where neutron fluences up to $10^{12} \mathrm{n} / \mathrm{cm}^{2}$ were integrated. UCM prototypes read out by the neutron irradiated SiPM boards were then tested at the CERN-PS T9 beamline. The calorimetric performance of the detector were not compromised by irradiation. During the beam test at CERN we were also able to perform the measurement of the photon-veto time resolution and we validated the 1-mip/2-mip separation capability using photon conversion from $\pi^{0}$ gammas.

\section{The ENUBET narrow band beam}

Assuming $4.5 \times 10^{19}$ POT at the CERN-SPS, about $1.13 \times 10^{6} v_{\mu}$-CC and $1.4 \times 10^{4} v_{e}$-CC interactions will be observed at a neutrino detector (500 $\mathrm{t}$ fiducial mass) located $50 \mathrm{~m}$ from the end of the tunnel. A narrow band beam such as ENUBET not only provides a beam with a precisely measured flux but also a measurement of the neutrino energy that does not rely on the reconstruction of final state particles. This feature ("narrow-band off-axis technique") results from the narrow momentum bandwidth of the beam and the finite transverse dimension of the neutrino detector. The energy measurement exploits the correlation between the energy of the neutrino interacting in the detector and the radial distance $(\mathrm{R})$ of the interaction vertex from the beam axis. The centroid of the 
distribution of the vertices of interacting neutrinos could be determined very well in a short time with a high-granularity detector like a LAr TPC ( $\mathrm{cm}$ level precision). The radial distance could then be measured on an event by event basis with an extremely good resolution. The incoming neutrino energy can be determined with a precision given by the pion peak width of the spectrum at a fixed $\mathrm{R}$. It ranges from $7 \%$ at $3.5 \mathrm{GeV}$ to $22 \%$ at $0.8 \mathrm{GeV}$.

\section{Acknowledgments}

This project has received funding from the European Research Council (ERC) under the European Unions Horizon 2020 research and innovation programme (grant agreement N. 681647).

\section{References}

[1] A. Longhin, L. Ludovici, F. Terranova, Eur. Phys. J. C 75 (2015) 155

[2] K. L. Brown etal., TRANSPORT, A Computer Program for Designing Charged Particle Beam Transport Systems, 1983, CERN-80-04

[3] T. J. Roberts and D. M. Kaplan, G4beamline simulation program for matter-dominated beamlines, 2007 IEEE Particle Accelerator Conference (PAC), Albuquerque, NM, (2007) 3468-3470

[4] A. Berra et al., CERN-SPSC-2016-036, SPSC-EOI-014, Geneva, 2014.

[5] Documentation available at http://enubet.pd.infn.it/.

[6] A. Berra etal., Nucl. Instrum. Meth. A 830 (2016) 345.

[7] A. Berra etal., IEEE Trans. Nucl. Sci. 64 (2017) 1056.

[8] G. Ballerini etal., JINST 13 (2018) P01028. 